\title{
Perceiving motion and rigid structure from optic flow: A combined weak-perspective and polar-perspective approach
}

\author{
MATS LIND \\ Uppsala University, Uppsala, Sweden
}

\begin{abstract}
Structure-from-motion algorithms based on weak-perspective projection have many interesting properties and could serve as a basis for a model of human perception of motion and structure from motion (M\&SFM). There is some psychophysical evidence, however, that points to discrepancies between what can be accomplished with these algorithms and the performance of human subjects in certain M\&SFM tasks. In light of this evidence, this paper presents a mechanism that both takes advantage of all the possibilities offered by a weak-perspective approach and behaves in a manner that is in close correspondence with human performance in M\&SFM tasks. It consists of a novel weak-perspectivebased method operating at small visual angles and a complementary, perspective-projection-based method operating at larger visual angles.
\end{abstract}

Relative motion between an object and a monocular observer has, in many cases, the potential of revealing the three-dimensional (3-D) structure of the object to the observer, along with parameters describing the relative motion. This circumstance has received much scientific attention, and there is, for instance, compelling empirical evidence that humans exploit this potential source of information about motion and 3-D structure (for an overview, see Rogers, 1993). A great number of theoretical and numerical studies (overviews are presented by Faugeras, 1993, pp. 245-300, and Maybank, 1993) have been published on how to make use of this potential source of information, in the following called motion and rigid structure from motion (M\&SFM). As a consequence, much is known about the nature of the problem and what can and cannot be accomplished by an M\&SFM process, whether implemented in a human-made system or in the functioning of a living creature.

One important factor in M\&SFM work is the type of projection relating one coordinate system (the 3-D world) to another (the projection surface). An ordinary camera, or the eye, is best described by what is known as perspective, or polar, projection. However, from a geometrical point of view, many different types of projections are conceivable. One example is orthographic projection, which has been used extensively throughout the history of M\&SFM research.

This work was supported in part by grants to Erik Börjesson from the Swedish Council for Research in the Humanities and Social Sciences. The author wishes to thank three anonymous reviewers and Erik Börjesson for their helpful comments. The author also thanks Andrew Zisserman, who provided data that helped to check the implementation of the Shapiro, Zisserman, and Brady algorithm. Correspondence should be addressed to M. Lind. Department of Psychology, Uppsala University, Box 1854, S-751 48 Uppsala, Sweden (e-mail: mats.lind@nomos.se).
Using different types of projection leads to different formulations of the M\&SFM problem and suggests different computational schemes to its solution. Recent theoretical work (e.g., Koenderink \& van Doorn, 1991; Shapiro, Zisserman, \& Brady, 1995) has shown that a particular projection type, often referred to as weak perspective, leads to formulations of the M\&SFM problem that have some interesting properties. For instance, the M\&SFM problem, in its weak-perspective form, can be robustly solved by a linear method even in the presence of measurement errors (Koenderink \& van Doorn, 1991; Shapiro et al., 1995). Algorithms based on weak perspective are, thus, of potential interest as a basis for models of the human M\&SFM process. There is some psychophysical evidence, however, that points to discrepancies between what can be accomplished by using these weakperspective approaches and the performance of human subjects in certain M\&SFM tasks. Therefore, the aim of this paper is to present a mechanism that both takes advantage of the possibilities offered by a weak-perspective approach and performs in close correspondence with human performance in M\&SFM tasks.

An overview of this paper is as follows: In Section 1, the concept of weak perspective is briefly described. The aim of Section 2 is to identify some important characteristics of the human M\&SFM process as it is reflected in the literature. In Section 3, a comparison is made between these characteristics and the performance of two recently published weak-perspective-based M\&SFM algorithms, and three discrepancies are identified. Section 4 contains the description, and some numerical evaluations, of a novel M\&SFM mechanism that is constructed to more closely match the performance of the human visual system when confronted with optic flow. In Section 5, the performance of this mechanism is compared with the results of studies on the perception of visual motion, especially motion 
patterns defined by the use of spatial derivatives of optic flow. Section 6 contains a brief discussion on some remaining issues.

\section{WEAK-PERSPECTIVE PROJECTION}

The physical projection in the human eye is best described through the use of perspective projection. Nevertheless, in certain cases, the physical projection in the eye can be approximated by another projection process. ${ }^{1}$ The most commonly used of these other projection processes has been orthographic projection. Orthographic projection is a reasonable approximation of perspective projection when a projected (viewed) object subtends a small visual angle, the internal depth of the object is small relative to the viewing distance, and the relative motion between the object and the projecting system contains no large component of motion directed toward or away from that system. Orthographic projection is used because it often leads to simple and effective solutions to M\&SFMrelated problems.

Weak-perspective projection can be seen as an elaboration of orthographic projection. They are identical in all aspects but one. If a projected object has a component of motion directed toward or away from the projecting system, when orthographic projection is used, the image of the object on the projection plane is left unchanged. However, when weak-perspective projection is used, the image is uniformly scaled (magnified or reduced). Because of this scaling, weak-perspective projection is also referred to as scaled orthographic projection.

When weak perspective is used, as in the case of orthographic projection, an M\&SFM process cannot recover all parameters of the relative motion between an object and the camera (the eye) from two 2-D views. One parameter will remain unknown until a third view is used. In leaving one parameter of relative motion unknown, the output from algorithms based on weak perspective, from two 2-D views, is a depth map of the "relief" kind (Koenderink \& van Doorn, 1991). However, unlike algorithms based on orthographic projection, they can, in many cases, estimate the psychologically interesting quantity "time to contact" (e.g., Lee, 1976; Tresilian, 1993).

Two M\&SFM algorithms for weak-perspective projection have recently been put forward: one by Koenderink and van Doorn (1991) and one by Shapiro et al. (1995). These algorithms show that the M\&SFM problem from two 2-D views can be solved linearly and robustly using least squares methods. An interesting detail in both of these algorithms is that they use a description of the relative motion between the camera (the eye) and the projected object that fits the descriptions given by human observers in similar conditions as reported by Börjesson and von Hofsten $(1973,1975)$. The results obtained by using a weak-perspective-based M\&SFM algorithm are illustrated in Figure 1.

\section{SOME CHARACTERISTICS OF THE HUMAN M\&SFM PROCESS}

To evaluate any M\&SFM algorithm in terms of its potential to serve as a basis for a model of the human M\&SFM process, the important properties of this process must be identified. The available psychophysical evidence is often too inconclusive or sparse to identify all of the relevant features of the human M\&SFM process. However, five areas can be identified that both have theoretical relevance and exhibit some useful psychophysical results. The first area concerns how to define the input to the process (i.e., what is the basis for the estimation of motion and structure in space from visual motion). The second area concerns how measurement errors could be dealt with. The third deals with the question of ad hoc assumptions. The fourth concerns the output of the process (i.e., the question of partial or complete solutions). The fifth relates to how incomplete input is handled.

\subsection{What Is the Input to the Human M\&SFM Process?}

2.1.1. Is time represented? The studies reported by McKee and Welch (1985) shed some light on this issue. Their results indicate that there are neural structures capable of producing estimates of local retinal velocities (i.e., time-related positional changes) and that the time frame used by these structures is in the range of $50-80 \mathrm{msec}$. Other results reported by Dosher, Landy, and Sperling (1989) and Landy, Dosher, Sperling, and Perkins (1991) show that the M\&SFM process in humans is impaired when the visual stimuli used are manipulated in ways known to impair the functioning of these velocity-sensing neural structures. Adopting these results, it seems reasonable to describe the input to the M\&SFM process as approximations to first derivatives of retinal position with respect to time (retinal velocities).

Apart from the psychophysical evidence, there are theoretical arguments favoring a view of retinal velocities as the basic input to the M\&SFM process. Perhaps the most important is related to the ultimate use of such a process. One of the probable purposes of recovering motion and structure in space is to support action (Gibson, 1966). If this is so, the time span used by the process must be short (Maybank, 1993, p. 119) and consistently applied.

Assuming that the input to the human M\&SFM process is retinal velocities has no bearing on how these velocities are sensed. The sensing could be based on graylevel operations or on time-related sequences of retinal coordinates. Furthermore, defining the input as retinal velocities does not mean that algorithms based on correspondences between image elements in successive views are disqualified as a basis for a model of the human M\&SFM process. On the contrary, it implies that velocity-based algorithms may also be used.

2.1.2. Dealing with input extended in time: One process or two? It is undoubtedly true that human per- 

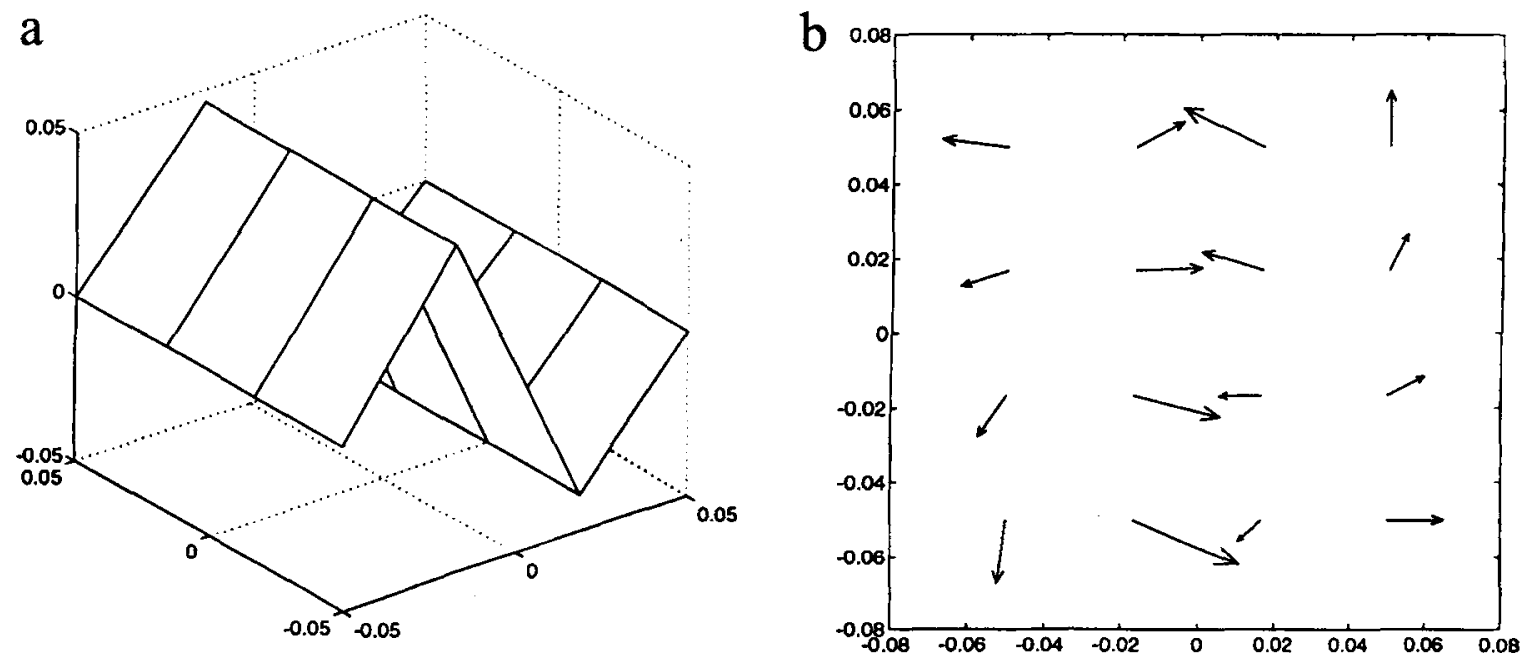

C

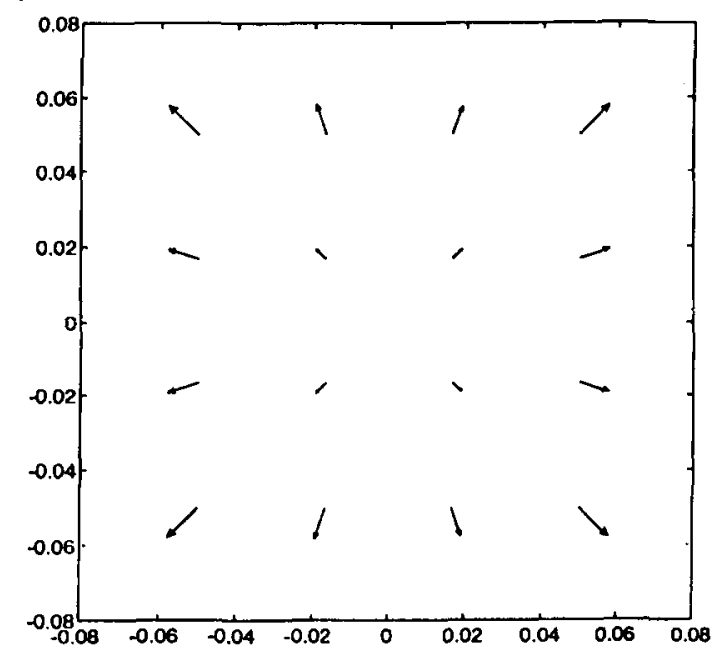

d

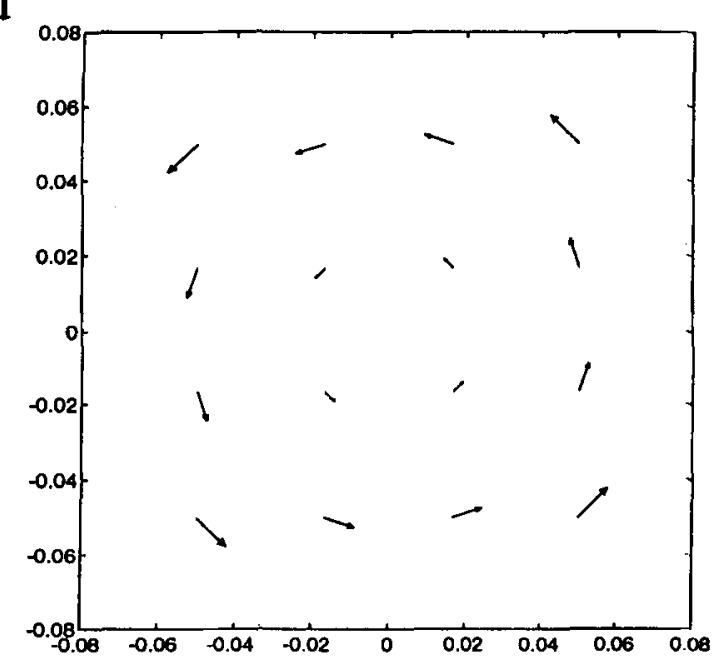

e

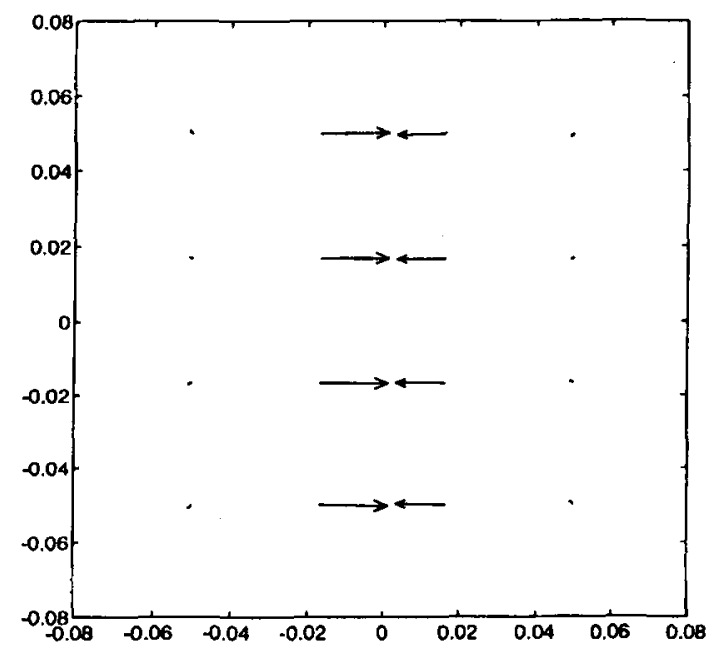

f

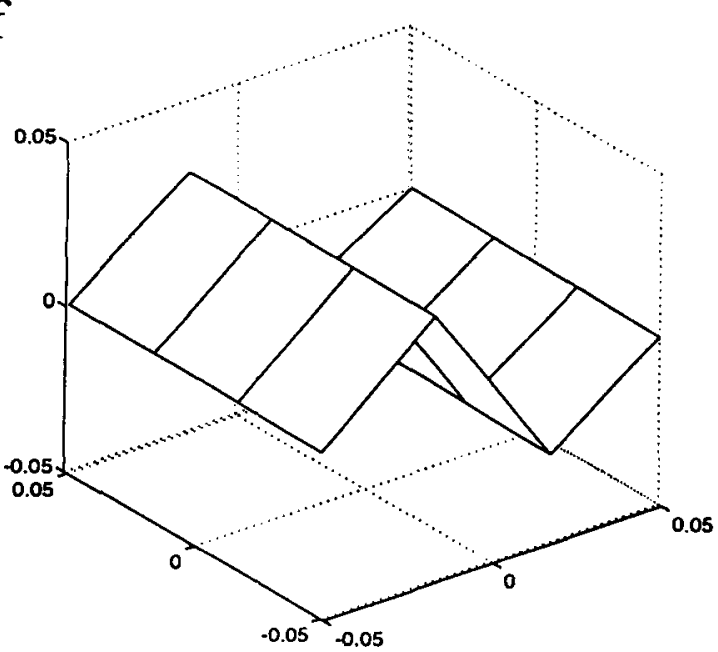

Figure 1. Illustration of performance of a weak-perspective-based M\&SFM algorithm. The algorithm actually used was the smallangle process described in Section 4. (a) Original synthetic 3-D object used. (b) A polar-projected flow field produced by moving the object and using the $z$-axis as the line of sight. (c) Translation of the object along the line of sight, as estimated by the process, displayed as 2-D vectors on the projection surface. (d) Rotation of the object around the line of sight, as estimated by the process, displayed as 2-D vectors on the projection surface. (e) Rotation around the axis in the $x y$ plane, as estimated by the process, including the errors produced by the polar (as opposed to weak-perspective) projection used. The result is displayed as 2-D vectors on the projection surface. (f) 3-D structure estimated by the process using the vectors shown in panel e. A randomly chosen value was used for the unknown rotational velocity (yielding an affine transformation). In this case, it led to a flattening of the original object. 
formance in many M\&SFM tasks grows better as the number of views is increased (e.g., Dosher et al., 1989; Husain, Treue, \& Andersen, 1989; Landy et al., 1991; Norman \& Todd, 1993; Todd \& Bressan, 1990; Todd \& Norman, 1991). It is important, however, to note that this could be due to at least two different kinds of processes. One kind of process assembles a sequence of sets of velocity estimates (three or more 2-D views) and performs the M\&SFM computation on the basis of the whole sequence. The other kind of process performs an M\&SFM computation on each consecutive set of velocity estimates (each consecutive set of two 2 -D views) and performs another computation on the resulting, likewise consecutive, 3-D information to obtain better information on true 3-D structure and relative motion. Both of these processes have been suggested in the machine vision literature, and it has been suggested that the analysis of successive 3-D representations can be accomplished by using Kalman filters (Faugeras, 1993; Shapiro et al., 1995).

There are psychophysical indications, although not decisive, that the two process view is a reasonable description of how the human visual system operates. Consider, for instance, the experiments reported by Husain et al. (1989). Their results suggest that the visual system uses a surface description of 3-D scenes defined by moving random dot stimuli instead of distance measurements to individual points. They reach this conclusion because their subjects' performance improved with more frames, even if all points were replaced by new ones every $100 \mathrm{msec}-$ given, of course, that all points belonged to the same rigid 3-D surface. As indicated by Husain et al. (1989), these results do not distinguish between surface descriptions in terms of 2-D velocity fields or 3-D surfaces. However, the results support a two-process view.

Although designed for a different purpose, studies by Todd and Bressan (1990), Todd and Norman (1991), and Norman and Todd (1993) indicate that there is a small effect of letting human observers see more than two views. However, they also clearly demonstrate that, given optimal conditions in terms of ISI and SOA (Todd, Akerstrom, Reichel, \& Hayes, 1988), two views are sufficient input to the human M\&SFM process for it to produce reliable 3-D percepts. This is further corroborated by the experiments performed by Börjesson and Lind (1996).

These results, viewed together, point to the distinct possibility that human behavior in M\&SFM tasks is governed by two separate processes. One, the basic M\&SFM process, operates on retinal velocities and produces a 3-D description. The other operates in 3-D on the time-varying characteristics of these 3-D descriptions.

In this paper, the two-process view is adopted, and the hypothesized basic process is considered. Consequently, the input to the process is defined to be retinal velocities (or, equivalently, two temporally close retinal views).

2.1.3. Retinal velocities as such or spatial derivatives of retinal velocities? The idea that spatial derivatives of a dense field of retinal velocities could serve as primitives in the recovery of motion and structure in space originates in the work of Koenderink and van Doorn
(1976). ${ }^{2}$ One of the main advantages of using first spatial derivatives ${ }^{3}$ is that the divergence and the deformation components describe properties of the relative motion and the 3-D layout that, at least during certain circumstances, could be used directly to guide behavior (see, e.g., Koenderink, 1986, 1990).

There are some pieces of psychophysical evidence, however, indicating that the human visual system does not use spatial derivatives as visual primitives. For instance, Braddick and Holliday (1992) investigated the "pop-out" potential of patterns exhibiting pure divergence or deformation. They concluded that the detection of these motion patterns, seen in a context of other motion patterns, could not be characterized as "popping out," but instead required serial search. This makes the existence of lowlevel mechanisms operating in parallel and sensitive to spatial derivatives unlikely. Furthermore, Nakayama and Tyler (1981) investigated the visual-motion-sensing system in terms of its filtering characteristics and concluded that it is best described as a low-pass spatial filter. This is also contraindicative to the visual system using spatial derivatives in that spatial derivatives imply the opposite (i.e., high-pass filtering). Taking these results into account, algorithms that could serve as a basis for the human M\&SFM process should be based on retinal velocities as such and not spatial derivatives of retinal velocities.

The results of the studies by Reagan and Beverley (1978), De Bruyn and Orban (1990, 1993), Freeman and Harris (1992), and Ahlström and Börjesson (in press) are also relevant to this question, but have even further implications and will be discussed in Section 5 .

\subsection{Handling Measurement Errors Is a Consequence of Using Retinal Velocities as Input}

An estimation of image velocities will usually contain measurement errors (Maybank, 1993). This is also confirmed in studies estimating the performance in this respect of the human visual system. Nakayama (1985), in citing several studies, summarizes these results as showing Weber fractions for retinal velocity of approximately $5 \%$.

One way of coping with this is to use techniques such as averaging or smoothing on the set of $2-D$ velocity vectors. This does not seem like a suitable technique for natural vision because of the problems it would lead to in the case of discontinuities in the set of retinal velocities with respect to position. Discontinuities of this kind are commonplace when an observer moves in natural environments. A typical example is locomotion through forests where rigidly connected objects, such as tree trunks and branches, with very different depth values often project onto neighboring parts on the retina. Another way of dealing with the problem of measurement errors is to use an error-reducing process, such as some kind of least squares process, in the recovery of the 3-D motion parameters only. This least squares strategy has been used in several M\&SFM algorithms (e.g., Maybank, 1993; Pradzny, 1980). To illustrate the rationale behind this strategy, consider the case of egomotion. 
During egomotion, as pointed out by Gibson (1950), every part of a rigid environment, regardless of its structure, contains information about the motion of the observer. Because of this, the more visual directions there are providing input to it, the better an error-reducing process estimating the egomotion works.

The distance from the observer to the environment, on the other hand, is unique in every visual direction, ${ }^{4}$ and nothing is gained when estimating distances from having input from more such directions.

Because the parameters of relative motion are identical in all visual directions in a rigid environment, a least squares solution that solves only for the parameters of relative motion will work. This approach has also been used in a number of previously published algorithms.

It is worth noting that when the parameters of relative motion are known, estimating the rigid 3-D structure is a fairly trivial matter. The estimated parameters of relative motion are simply used in conjunction with each velocity vector. This directly yields the relative distance to the distal point associated with the vector.

\subsection{No Ad Hoc Assumptions About the Nature of the Relative Motion Are Viable}

There are a number of algorithms that work only under the assumption that one or more of the parameters of relative motion is known before the analysis begins-for instance, that the axis of rotation is known or that the observer is engaged in a pure translatory motion in relation to the environment. Although such algorithms can provide valuable insight into the human M\&SFM process and serve as theoretical background in empirical work, they do not seem to be likely candidates as descriptions of the human M\&SFM process as a whole.

Perhaps the most common assumption of this kind is hidden in the usage of pure orthographic projection. If pure orthographic projection is to be a justifiable simplification, it must be assumed that the average distance between the eye and the viewed distal points is unchanged by the relative motion. However, the sensitivity of the human visual system to 2-D expansions and contractions (e.g., Reagan \& Beverley, 1978) and the empirical data on the perception of time to contact (for an overview see Tresilian, 1993) indicate that this is not a reasonable assumption when modeling human vision.

\subsection{Complete or Partial Solutions}

An inherent feature of any optic-flow (see Section 4.1)-based M\&SFM algorithm based on either weakperspective or orthographic projection is its inability to estimate the amount of rotation of a viewed object around a rotational axis orthogonal to the line of sight. This results in a depth map consisting of a one-parameter family of depths (Koenderink \& van Doorn, 1991) sometimes referred to as a relief..$^{5}$ An algorithm based on perspective projection does not share this inability. It can, at least in principle, estimate all parameters of motion from optic flow given an input that is adequately varied. This aspect of M\&SFM algorithms based on perspective projection has been investigated by Koenderink and van Doorn (1987). Both their theoretical analyses and their numerical simulations show that, in the presence of measurement errors, the precision in the estimation of the critical parameter of relative motion is a positive function of visual angle and, to a somewhat lesser extent, the number of landmarks.

These results point to a way of assessing whether the M\&SFM process of the human visual system is based totally on the simplifications offered by assuming weakperspective projection or whether, given wide angle input, it uses the full information available in perspective projection. Several experiments have been performed by Börjesson and Lind (1996) and Lind and Börjesson (1996) in an attempt to empirically investigate this. The results are clear. The consistency by which human subjects estimate the parameter of relative motion left unknown by a weak-perspective process is a function of visual angle as predicted by the Koenderink and van Doorn (1987) findings. However, although this was true for the consistency - that is, each subject estimated the value of the parameter in a way which was directly related to, and only related to, the actual value of it--the predictions did not hold for the actual value assigned to the parameter. When the visual angle was small, the distances estimated by the subjects varied between subjects and were also inconsistently related to the actual distances. This can be interpreted as due to the difficulties involved in estimating all parameters of relative motion at small visual angles. When the visual angle was large, the distances estimated by the subjects varied only between subjects but were consistent with actual distances for each subject.

These results have some interesting implications on the "euclidean versus affine" issue (see, e.g., Koenderink \& van Doorn, 1991; Lappin \& Ahlström, 1994; Lappin \& Love, 1992; Norman \& Todd, 1993; Pizlo \& SalachGolyska, 1994; Todd \& Bressan, 1990; Todd \& Norman, 1991). However, in the present context, all that matters is that all parameters of motion indeed were estimated by the subjects at large visual angles from only two views, although at least one of them was scaled by a subjectspecific factor. This cannot be accomplished by an M\&SFM algorithm based on weak-perspective or orthographic projection analyzing retinal velocities.

When evaluating M\&SFM algorithms in terms of their potential to serve as a basis for a model of human perception of motion and structure from retinal velocities, it seems (because of the above) reasonable to assume that such algorithms should be able to output complete or partial solutions depending on the nature of the input. If the visual angle is large and the viewed scene is, for the most part, rigid (as is usually the case during locomotion), the output should be a solution where all parameters of relative motion are estimated. If any or both of these conditions are violated, different kinds of partial solutions should constitute the output.

\subsection{Handling Incomplete Input}

Börjesson and von Hofsten $(1973,1975)$ report that three points engaged in relative motion are, at least in 
many instances, capable of eliciting stable 3-D percepts in observers. As is clear from theory, these impressions cannot, in the case of general motion of a 3-D object viewed by the human eye, be veridical because four noncoplanar points are the theoretical minimum if a relief depth map is to be calculated (e.g., Koenderink \& van Doorn, 1991); five noncoplanar points are the minimum if a complete output is considered (e.g., Maybank, 1993). However, because the percepts seem to be consistent for each type of 2-D motion pattern over subjects and time, it seems probable that the human M\&SFM process is capable of applying additional constraints in the case of incomplete input.

Thus, another desirable quality of an algorithm suitable as a basis for the human M\&SFM process is that it be capable of easily incorporating additional constraints.

\section{WEAK-PERSPECTIVE M\&SFM ALGORITHMS AS MODELS OF THE HUMAN M\&SFM PROCESS}

The weak-perspective M\&SFM algorithms by Koenderink and van Doorn (1991) and Shapiro et al. (1995) match several of the characteristics listed in the previous section. They can use the described input, they are robust in the presence of noise, and they make no ad hoc assumptions on the nature of the relative motion. They also have computational advantages in that they employ linear least square methods.

In light of the criteria described in Section 2 of this paper, however, these algorithms have three weaknesses. First, they can never estimate all parameters of relative motion from retinal velocities, no matter how large a visual angle the viewed environment subtends. Second, a basic assumption of both of these algorithms is the existence of at least four noncoplanar points. If the viewed object is represented by three points (or, equivalently, is planar), this is discovered, but no other useful information about the viewed object or the relative motion can be extracted by these algorithms. Third, they cannot handle the case where the relative motion between an object and an observer can be described as motion purely along and/ or around the line of sight. This case cannot be distinguished by these algorithms from the case of an arbitrarily moving planar object. In both cases, the algorithms only signal that the input is incomplete.

Of these three weaknesses, only the first is a result of the choice of weak perspective as the projection process. To understand the existence of the other two, it must be kept in mind that these algorithms primarily are designed to recover structure from motion (SFM) and not M\&SFM.

\section{YET ANOTHER M\&SFM MECHANISM}

\subsection{Defining Optic Flow}

As is clear from Section 2, the input to a mechanism relevant to the basic human M\&SFM process should be the 2-D information available in two temporally close views. A useful concept in light of this is "optic flow," and, to define it, the Koenderink and van Doorn (1987) terminology is adapted. Consequently, the environment is considered to contain "landmarks that can be recognized for the duration of a short span of time and thus serve as markers for the optic flow" (Koenderink \& van Doorn, 1987, p. 247). Furthermore, a projection point and the environment move relative to one another. Rigidly connected to the projection point is a projection surface. The projections of the landmarks on the projection surface, in terms of positions and velocity vectors, are considered to constitute the optic flow. Thus, the flow is "specified through a finite number of vectors only, and not through a continuous field" (Koenderink \& van Doorn, 1987, p. 247). ${ }^{6}$

\subsection{Frame of Reference}

From a computational view, it makes no difference whether the case of observer motion relative to an environment is considered or whether the case considered is of a stationary observer viewing a moving object. It suffices to consider the relative motion between the observer and the object. In fact, it makes good sense to distinguish between two computational levels. The first level involves determining the 3-D structure of the object and the relative motion between the observer and the object regardless of what gave rise to this relative motion. The second level involves determining whether this relative motion is due to object motion, ego-motion, or both.

This paper considers the first level- that is, the determination of the relative motion and the structure of the viewed scene. (This position taken, it is still worth mentioning that the source of the relative motion has some important secondary effects. Ego-motion, for instance, almost always produces wide-angle input to the M\&SFM process, whereas object motion almost never does so.)

Following Koenderink and van Doorn (1991) and Shapiro et al. (1995), and as a matter of computational convenience, a view of the relative motion as object motion is adopted. As can be understood from the preceding discussion, this does not have any significance on the second level of analysis - that is, it does not ascribe this relative motion to actual object motion, ego-motion, or both.

The origin of the coordinate system used is positioned, at $t=t_{0}$, on a landmark of the viewed object, and, as a function of time, this landmark is allowed to slide on the $z$-axis. In practice, this can be accomplished by a visual system actually fixating a point on the object, or it can be done by means of a computation on an arbitrarily produced flow field. This procedure reduces the number of unknowns to be solved by making parts of the rotational velocities dependent on the translational component of the fixated point and can thus be conceived of as a kind of "preprocessing."

\subsection{Describing the Relative Motion Between a Rigid Object and the Frame of Reference}

The relative motion between a rigid object and the system can be described in terms of the following components (see Figure 2): (1) translation of the object along the $z$-axis (one parameter: $\dot{z}$, a velocity); (2) rotation of 


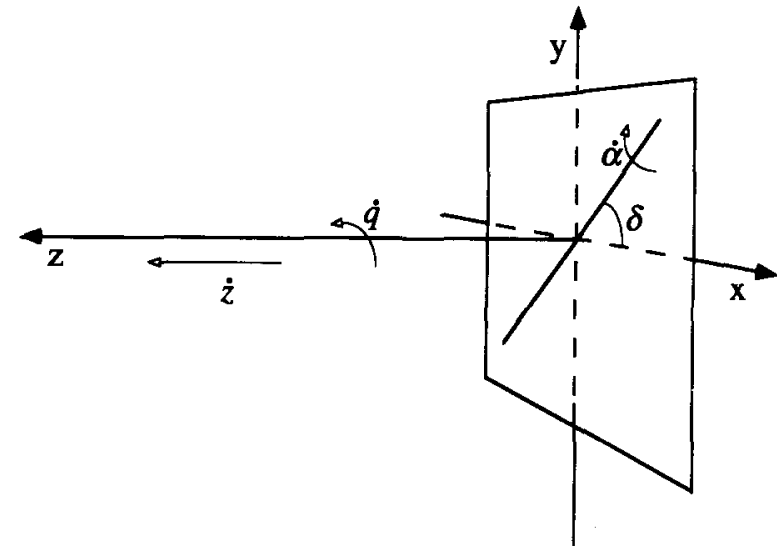

Figure 2. The parameters describing the relative motion.

the object around the $z$-axis (one parameter: $\dot{q}$, a rotational velocity); and (3) rotation of the object around an axis through the fixated point and orthogonal to the $z$-axis (two parameters: $\delta$, the angle between this axis and the $x z$ plane, and $\dot{\alpha}$, the rotational velocity around this axis). The rotational axis described by $\delta$ will be referred to as the $x y$ rotational axis.

This description of relative motion is similar to the one used by Koenderink and van Doorn, (1991) and Shapiro et al. (1995). There are two main advantages to this description. First, when the visual angle is small and partial solutions are found, these solutions are easy to interpret (Koenderink \& van Doorn 1991; Shapiro et al., 1995). Second, it fits subjects' phenomenological experiences when viewing moving dot patterns (Börjesson \& von Hofsten, 1973, 1975).

\subsection{The Projection Process}

The $x y$ plane of the coordinate system is used as the projection surface and the projection point is positioned on the $z$-axis at the point $z=1$. This convention can be used because only relative (as opposed to absolute) distances are recovered by any M\&SFM process. If the distance to the fixation point can be estimated using other processes, the absolute distances can be estimated simply through multiplying each $z$-coordinate (recovered by the M\&SFM process) by the estimated distance to the fixation point.

\subsection{Components of Motion and Their Projections}

In the following, "retinal" coordinates and their derivatives with respect to time will be used, rather than a more compact notation based on matrices. The reason for this is primarily a desire to follow a long tradition within the psychological literature on the M\&SFM problem.

The mathematical delineation of the equations is presented in Appendix A. It should be noted that the distance to each point in these equations is described not in terms of its $z$-coordinate directly but in terms of the distance $D . D$ is a direct function of the $z$-coordinate, such that $D=Z /(1-Z)$. Both $Z$ and $D$ are treated in the equations, explicitly or implicitly, as functions of retinal loca- tion - that is, $D(x, y)$ and $Z(x, y)$. Using $D$ as an unknown instead of $Z$ simplifies the calculations somewhat. When $D$ is found, it is easy to determine $Z[Z=D /(1+D)]$. Throughout the presentation of the equations, the index representing the point in question is dropped for better readability except when needed for clarity.

4.5.1. Rotation around the $z$-axis. This component of relative motion produces vectors that are independent of the 3-D layout of the viewed scene. The direction and the magnitude of each vector is dependent solely on the position on the projection plane and the magnitude of the rotation. The equations in terms of $\dot{x}$ and $\dot{y}$ are

$$
\dot{x}=-y \dot{q}
$$

and

$$
\dot{y}=x \dot{q} .
$$

4.5.2. Translation of the projection point along the $z$-axis. This component of relative motion produces vectors that are all directed along the line connecting, on the projection surface, the origin and the point in question. The magnitudes of the vectors are determined, except for the obvious influence of $\dot{z}$, in part by the distance to the origin on the projection surface and in part by the 3-D layout. If $D$ is close to zero - that is, small compared with the distance between the projection point and the point on the object being fixated (this distance is always considered to be 1 at $t=t_{0}$ ) - 3-D layout is unimportant, and the vectors are largely determined by the translational velocity and position of the viewed point on the projection surface. (This is exactly the simplification, with regards to the translation of the object along the $z$-axis, used by a weak-perspective projection process, the projection process employed by Koenderink \& van Doorn, 1991, and by Shapiro et al., 1995; cf. Section 1.)

The equations in terms of $\dot{x}$ and $\dot{y}$ are

$$
\dot{x}=x \dot{z}+x \dot{z} D
$$

and

$$
\dot{y}=y \dot{z}+y \dot{z} D .
$$

4.5.3. Rotation of the distal object around an axis in the $x y$ plane. For reasons that will become apparent later, the effects of this component of motion are described presupposing that the axis around which the object is rotating is the $y$-axis of the coordinate system (i.e., letting $\delta=90^{\circ}$ ). Using this presumption, the equations in terms of $\dot{x}$ and $\dot{y}$ are

$$
\dot{x}=x^{2} \dot{\alpha}-\dot{\alpha} D
$$

and

$$
\dot{y}=x y \dot{\alpha} .
$$

It is worth noting that the projected $y$-velocity component of the object's rotation around the $y$-axis (Equation 6) is independent of the object's 3-D structure. This is easily understood if this component of motion is viewed in terms of ego-motion instead of object motion. In the case of ego-motion, this component of motion can be interpreted as a sum of a translational velocity com- 
ponent along the $x$-axis (and thus orthogonal to the line of sight) and a rotation of the optical system to keep the projected velocity of the fixated point zero. Because the vectors caused by a translation of the optical system always are parallel if the translation is orthogonal to the line of sight, only the rotation of the optical system produces $y$-components. Velocity components produced by a rotation of the optical system are always independent of the 3-D structure of the viewed object. Thus, the projected $y$-velocity component of the object's rotation around the $y$-axis is independent of the object's 3-D structure.

4.5.4. Any motion. The velocity vector components of a point on the projection surface are simply the sum of the above-mentioned vector components. Still presupposing that the $y$-axis is the axis around which the object is rotating, the combined equations in terms of $\dot{x}$ and $\dot{y}$ are

$$
\dot{x}=x^{2} \dot{\alpha}-\dot{\alpha} D+x \dot{z}+x \dot{z} D-y \dot{q}
$$

and

$$
y=x y \dot{\alpha}+y \dot{z}+y \dot{z} D+x \dot{q} .
$$

\subsection{Small Visual Angles}

4.6.1. Weak perspective as an approximation of polar projection. A weak-perspective approach is used in the case of small visual angles. However, the relevant equations will first be formulated as approximations to polar projection instead of directly using weak perspective. This is done to illustrate the relationship between the two projection types.

When examining Equations 7 and 8, it becomes apparent that the terms $x^{2} \dot{\alpha}$ in Equation 7 and $x y \dot{\alpha}$ in Equation 8 will be difficult to distinguish from noise generated in the measurement process when the visual angle is small. This is due to the fact that, given the definitions used in this paper, small visual angles mean that all $x$ and $y$ values are much smaller than 1 . The product of two such values is only a small fraction of the values themselves.

Therefore, the terms $x^{2} \dot{\alpha}$ and $x y \dot{\alpha}$ can be disregarded at small visual angles, and Equations 7 and 8 can be written as

$$
\dot{x} \approx-\dot{\alpha} D+x \dot{z}+x \dot{z} D-y \dot{q}
$$

and

$$
\dot{y} \approx y \dot{z}+y \dot{z} D+x \dot{q} .
$$

These approximations are also inherent in the use of orthographic (and, consequently, weak-perspective) projection when delineating the effects on $\dot{y}$ and $\dot{x}$ of the rotation of the distal object around the $x y$ rotational axis (still presupposing that $\delta=90^{\circ}$ ).

To further simplify these equations, one more approximation inherent in the use of weak perspective can be utilized - namely, to consider the effect of the translational velocity along the $z$-axis (on $\dot{y}$ and $\dot{x}$ ) to be expressed by $y \dot{z}$ and $x \dot{z}$ only. Equations 7 and 8 can then, at small visual angles, be written

$$
\dot{x} \approx-\dot{\alpha} D+x \dot{z}-y \dot{q}
$$

and

$$
\dot{y} \approx y \dot{z}+x \dot{q} .
$$

As a comparison, using weak perspective directly yields

$$
\dot{x}=-\dot{\alpha} Z+x \dot{z}-y \dot{q}
$$

and

$$
\dot{y}=y \dot{z}+x \dot{q} .
$$

These equations mean that when the $y$-axis of the coordinate system is oriented along the $x y$ rotational axis of the distal object, the $\dot{y}$ values can be described as a linear combination of image coordinates. Geometrically, this can be interpreted as a planar surface in $(x, y, \dot{y})$ space, which passes through the origin. (When polar projection is used, this surface will only be approximately planar.) The orientation of the plane is determined by the two parameters of relative motion $\dot{z}$ and $\dot{q}$ and is thus unaffected by the 3-D structure of the viewed object.

If the viewed object is planar-that is, $Z(x, y)=a x+$ $b y$ - the $\dot{x}$ values can also be described by a linear combination of image coordinates, in that $\dot{x}=(\dot{q}-a \dot{\alpha}) x+$ $(\dot{z}-b \dot{\alpha}) y$. However, if the landmarks of the viewed object are noncoplanar, the $\dot{x}$ values can no longer be described as such a combination. This circumstance can be used to define an M\&SFM method that has some interesting properties in relation to human perception of M\&SFM.

4.6.2. The first step of the small-angle method. If the direction of the $x y$ rotational axis (the value of $\delta$ ) is known, the coordinate system on the projection surface can be rotated such that the $y$-axis is coincident with the $x y$ rotational axis, and the best fitting plane, in a least square sense, in $(x, y, \dot{y})$ space can then easily be determined. The parameters of such a plane can, for instance, be recovered by using the "normal equations" for multiple regression with two unknowns, directly on the measured quantities. Thus,

$$
\dot{z}_{e s t}=\frac{\sum_{i=1} x_{i} y_{i} \sum_{i=1} x_{i} \dot{y}_{i}-\sum_{i=1} x_{i}^{2} \sum_{i=1} y_{i} \dot{y}_{i}}{\left[\left(\sum_{i=1} x_{i} y_{i}\right)^{2}-\sum_{i=1} x_{i}^{2} \sum_{i=1} y_{i}^{2}\right]}
$$

and

$$
\dot{q}_{e s t}=\frac{\sum_{i=1} y_{i} \dot{y}_{i}-\dot{z}_{e s t} \sum_{i=1} y_{i}^{2}}{\sum_{i=1} x_{i} y_{i}} .
$$

The coefficients found will be estimates of the parameters $\dot{z}$ and $\dot{q}$. To get an estimate of how well the found plane describes the actual data, the root of the mean square of the residuals can be calculated. That is,

$$
f=\sqrt{\frac{\sum_{i=1}^{n}\left(\dot{y}_{i}-\dot{y}_{p_{i}}\right)^{2}}{n}},
$$

where $f$ is the measure of the error of the fit, $\dot{y}_{p}$ the value predicted by using $\dot{z}_{\text {est }}$ and $\dot{q}_{\text {est }}$ in Equation $8 \mathrm{wp}$, and $n$ is the number of points used in the estimation. A low value of $f$ indicates a good fit. 
Next, consider a slight rotation of the coordinate system away from the $x y$ rotational axis of the distal object. The $y$-axis will no longer exactly coincide with the $x y$ rotational axis, and the $\dot{y}$ values in this new system will be influenced by $\dot{x}$ values of the original coordinate system and, thus, the 3-D structure of the viewed object. The new $\dot{y}$ values are described by

$$
\dot{y}^{\prime}=y^{\prime} \dot{z}+x^{\prime} \dot{q}+\dot{\alpha} Z\left(x^{\prime}, y^{\prime}\right) \sin \gamma,
$$

where $\gamma$ is the angle between the $x y$ rotational axis and the $y$-axis of the system. If, as before, a regression is performed using Equation 8wp, the value of $f$ will be higher than the value found when performing the regression in the original coordinate system, unless, of course, the viewed object is planar.

In the general case, the direction of the distal $x y$ rotational axis is not known. Nevertheless, its direction can, following the above, be determined by finding the direction of the $y$-axis of a rotated coordinate system on the projection surface that has the lowest value of $f$. From a strictly computational point of view, a gradient-based iterative method could be used to find the direction that produces the lowest value of $f$. However, this would only lead to a weak-perspective M\&SFM algorithm that suffers from the same shortcomings, described in Section 3, as do the algorithms proposed by Koenderink and van Dorn (1991) and Shapiro et al. (1995). Furthermore, it would solve the problem by using iterative search where robust linear methods are possible (Shapiro et al., 1995).

Instead, a similar result can be obtained by another method that does not exhibit the aforementioned shortcomings except the one inherent in the choice of weakperspective projection as such. The method is based on the fact that the orientation of the unknown $x y$ rotational axis of the distal object can be expressed as a direction on the projection surface. It is therefore possible to have a number of coordinate systems defined on the projection surface each rotated differently in relation to the frame of reference used (see Figure 3). In every coordinate system, a regression using Equation 8 wp could be performed. The combined output of these parallel regressions has some interesting properties.

Before these are discussed, however, the first part of the proposed method for small visual angles is described: (1) A set of Cartesian coordinate systems on the projection surface is defined. The systems have a common origin, but each system has its $y$-axis in a unique direction. (For this method to function in the general case, the whole possible range of $180^{\circ}$ should be covered by the coordinate systems.) The number of systems in the set and their respective orientations determine the accuracy of the process. (2) The velocity vectors on the projection surface are projected onto the $y$-axis of each of these systems, and sets of $\dot{y}, x$, and $y$ values are formed. One such set is formed per coordinate system. (3) A regression is performed in each coordinate system using these sets. The output of each regression is the $f$ value and the estimated values of $\dot{z}$ and $\dot{q}$.

The input to the method cannot be assumed to be free of measurement errors (see Section 2.2). The value of $f$
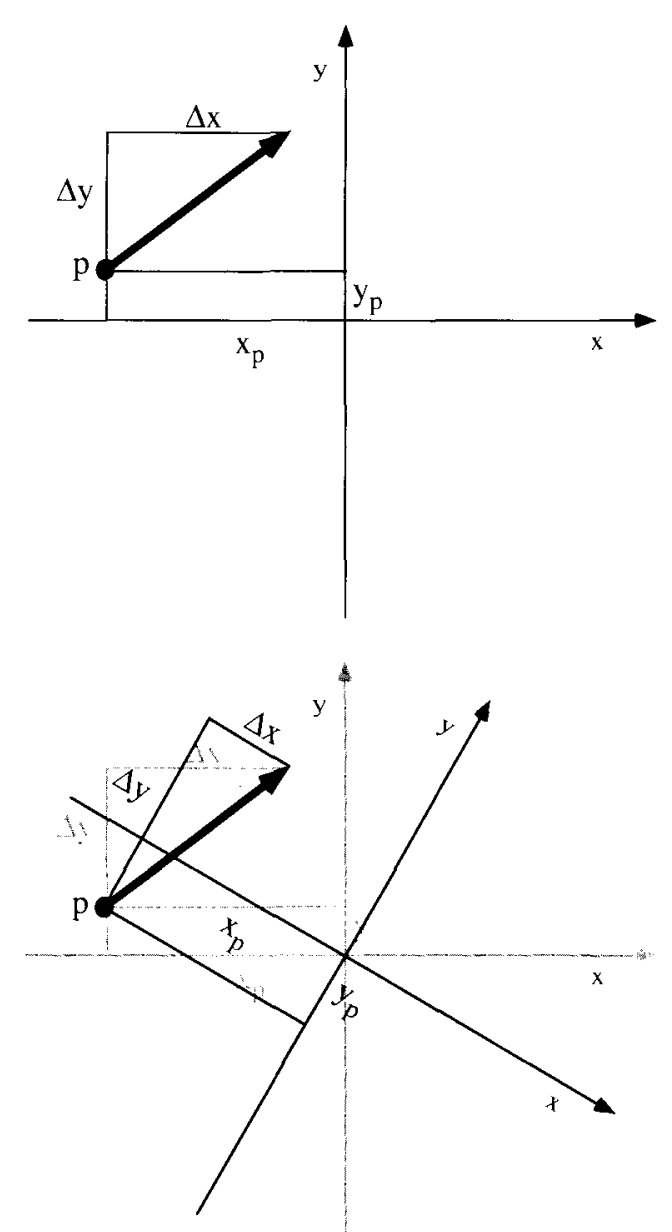

Figure 3. The upper figure illustrates a 2-D frame of reference on the projection surface and a point, $p$, together with a velocity vector associated with $p$. The lower figure illustrates a coordinate system that is rotated with respect to the original frame of reference and how the description of the point $p$ and its associated velocity vector is changed with the rotation.

can thus never be expected to be zero, not even for a coordinate system having a $y$-axis exactly coinciding with the $x y$ rotational axis. However, an expected minimum value will exist that is related to the level of noise in the measuring system. This expected minimum value of $f$ will be referred to as the system noise level.

4.6.3. The second step of the small angle method. The next step is to evaluate the combined output from the differently rotated coordinate systems. This can be described in terms of three easily identifiable output patterns and their interpretations in terms of distal events.

The most potent of these output patterns occurs when one of the coordinate systems (or two adjacent systemsin which case, either system can be chosen) has a value of $f$ that is the lowest, and this value is close to the system noise level. Such a pattern is formed by at least four noncoplanar landmarks that are engaged in rigid relative motion containing a nonzero component of rotation around an $x y$ rotational axis (i.e., this output pattern corresponds 
to situations where the Shapiro et al., 1995, or the Koenderink \& van Doorn, 1991, algorithms are applicable). The $y$-axis of the coordinate system with the lowest $f$ value is showing (with a resolution related to the number of systems used) the direction of the distal $x y$ rotational axis, and the $\dot{z}$ and $\dot{q}$ values from the regression are good estimates of the real $\dot{z}$ and $\dot{q}$ values. These estimated values can be used in conjunction with the measured $\dot{y}$ values in the coordinate system located orthogonally to the system having the lowest $f$ value (i.e., the $\dot{x}$ values) to calculate the distances $\dot{\alpha} D(x, y)$ using Equation $7 \mathrm{~b}$. The analysis in this case gives solutions to the M\&SFM problem that are very similar to the solutions obtained with the algorithm described by Shapiro et al. (1995) (see Section 4). The process that finds the minimum $f$ value can be simple in that the coordinate systems can be implemented as physical structures operating in parallel.

Another distinctive pattern occurs when all coordinate systems show a value of $f$ that is close to the system noise level, but the estimated values of $\dot{z}$ and $\dot{q}$ differ between the systems. This pattern occurs when the viewed landmarks are engaged in rigid relative motion containing a nonzero component of rotation around an $x y$ rotational axis, are coplanar (or, equivalently, when the pattern consists of only three landmarks) but not colinear, and the plane defined by the landmarks is nonorthogonal to the line of sight. When this output pattern occurs, at least two things can be done. One is to apply an additional constraint, for instance, that the rotational component around the line of sight should be as small as possible (Koenderink, 1990). This is simple to implement in that the $\dot{q}$ (or $\dot{z}$ ) values from the different directions can be compared in the same manner as the $f$ values. The other option is to deliver the estimates in all directions to the next perceptual level-in effect, showing the solution space.

A third important pattern occurs when all coordinate systems show $f$ values close to the system noise level, and they all also show identical (within the boundaries set by the noise level) $\dot{z}$ and $\dot{q}$ values. This pattern occurs, with one exception, when the relative motion of the viewed landmarks is rigid and is taking place only along and/or around the line of sight. The estimated values of $\dot{z}$ and $\dot{q}$ are close to the true $\dot{z}$ and $\dot{q}$ values, and this is true even for coplanar landmarks (including the case of only three noncolinear landmarks). ${ }^{7}$ The exception mentioned above is when the viewed object is planar and orthogonal to the line of sight. In this case, the estimated $\dot{z}$ and $\dot{q}$ are, as before, close to the true $\dot{z}$ and $\dot{q}$ values; however, a rotation around an $x y$ rotational axis might exist. If it does, however, it will be evident at later moments, because the planar configuration of landmarks will then no longer be orthogonal to the line of sight (it will have been rotated by the nonzero component of rotation around the $x y$ rotational axis), and the estimations of $\dot{z}$ and $\dot{q}$ will start to differ between the coordinate systems.

These three output patterns and their proposed interpretations, viewed as an M\&SFM method, do not suffer from the shortcomings discussed in Section 3, except for the one inherent in the use of weak perspective as such. Hence, (1) when the input is rigid and complete, results similar to the results recovered by the algorithm proposed by Shapiro et al. (1995) are obtained, (2) when the input is rigid but incomplete, additional constraints can easily be applied or the solution space can be presented to higher perceptual or cognitive mechanisms, and (3) when the input is created by rigid objects moving only along and/or around the line of sight, this is perceived and the relative motion is extracted.

The small-angle method is illustrated in Figure 4.

4.6.4. Numerical evaluation of the small-angle method. The quality of the output will depend on the number and the distribution of the differently rotated coordinate system used and the appropriateness of the proposed error function. To evaluate the latter, a large number (27,000 runs) of numerical comparisons to the Shapiro et al. algorithm have been made using polar-projected synthetic optic-flow fields produced by the use of simulated rigidly moving, noncoplanar landmarks. The two error functions give very similar results in all conditions. Some examples of the results are presented in Figure 5. The details of how the numerical simulations were performed are described in Appendix B.

An interesting feature of both these error functions can be seen in Figure 6. As the visual angle is increased during the conditions tested, the error in the estimate of the direction of the $x y$ rotational axis increases, but, at the same time, the error in the estimates of the two velocities $\dot{z}$ and $\dot{q}$ decreases. This could point to the existence of an optimal size, in terms of visual angle, within which the small-angle algorithm should operate.

\subsection{Large Visual Angles}

4.7.1. A wide-angle process yielding a relief depth map. If, for some reason, a depth map of the relief kindis desirable over the whole field of view using polarprojected input, this can be accomplished within the present framework. This is best described by first assuming that the rotation around the $z$-axis is zero and that the $x y$ rotational axis of the object is the $y$-axis. Equations 7 and 8 can then be written

$$
\frac{\dot{x}}{x}=x \dot{\alpha}-\frac{\dot{\alpha} D}{x}+\dot{z}+D \dot{z}
$$

and

$$
\frac{\dot{y}}{y}=x \dot{\alpha}+\dot{z}+\dot{z} D .
$$

Because all terms in these two equations are identical except for the $-\dot{\alpha} D / x$, this can be expressed as

$$
\frac{\dot{y}}{y}-\frac{\dot{x}}{x}=\frac{\dot{\alpha} D}{x} .
$$

If the variables are expressed in terms of polar coordinates $(r, \theta)$ and their derivatives with respect to time, this equation can in turn be written as 
a

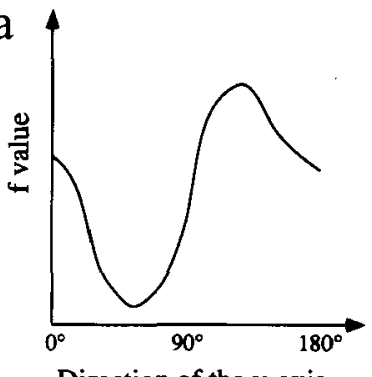

Direction of the y-axis
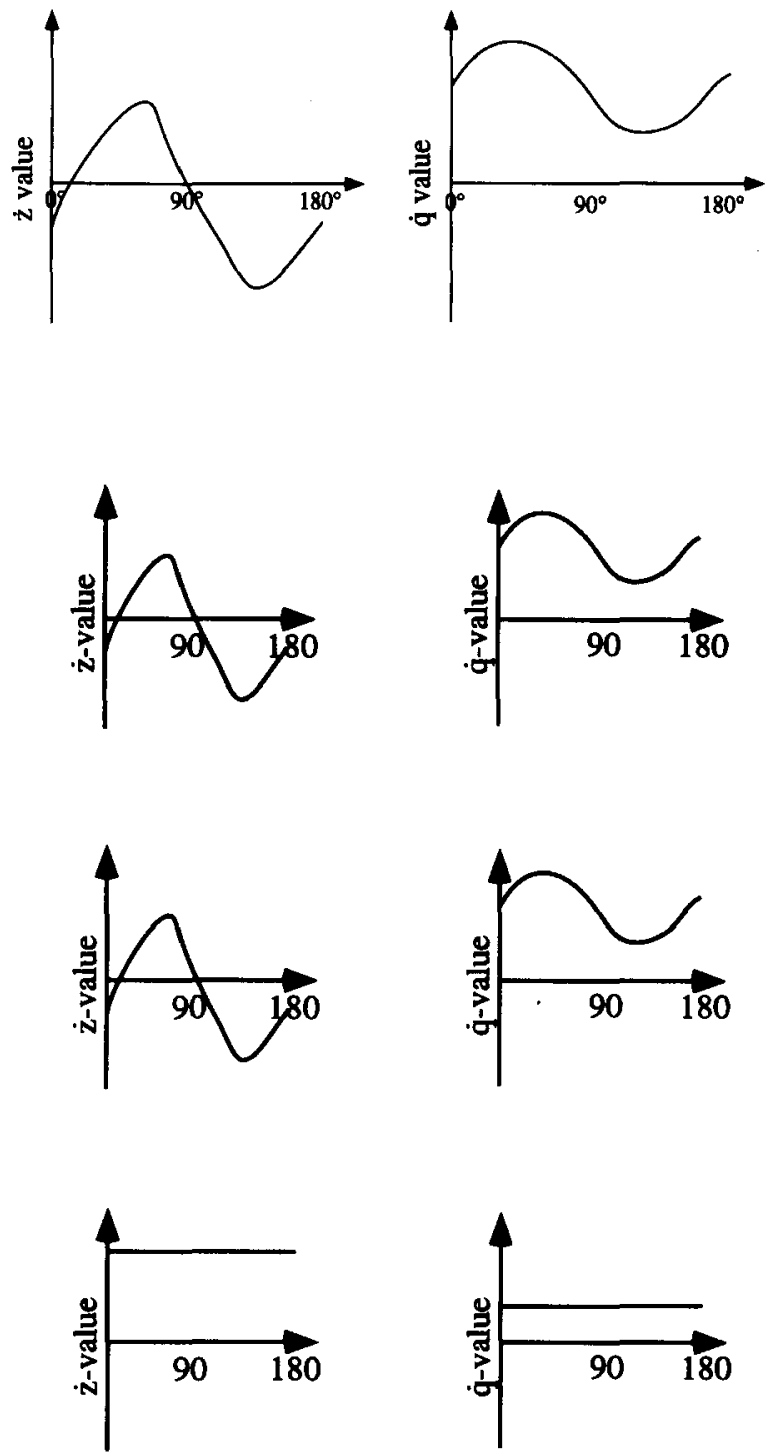
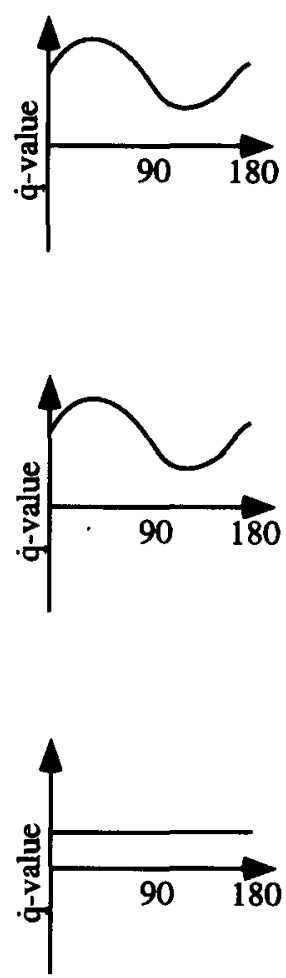

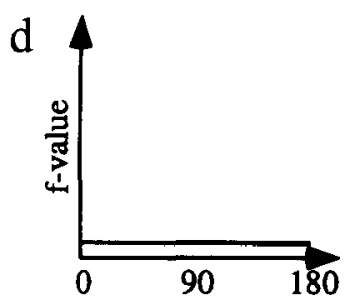

Direction of the $y$-axis

Figure 4. (a) Example of output from the first part of the small-angle process. (b) One of the main three output patterns of the small-angle process. The proposed method interprets it as caused by a rigid and noncoplanar constellation of at least four points. The direction of the $x y$ rotational axis is given by the direction having the smallest value of $f$. This direction then determines the $\dot{z}$ and $\dot{q}$ values. (c) Another of the three main output patterns. The proposed method interprets it as caused by a rigid and planar constellation of three or more points. An $x y$ rotational axis exists but cannot be determined without additional information or constraints. (d) The third of the three main output patterns. If this pattern persists over time, the proposed method interprets it as caused by a rigid constellation of points translating along and rotating about the line of sight. The $\dot{z}$ and $\dot{q}$ values are recovered.

$$
\frac{r \dot{\theta}}{\sin (\theta)}=\dot{\alpha} D
$$

The quantities $\dot{\alpha} D_{n}$ provide the desired depth map of the relief kind.

The general case of unknown motion can now be described. In Equation 12, both $r$ and $\dot{\theta}$ are independent of the direction of the axes of the associated 2-D Cartesian coordinate system on the projection plane. Thus, these quantities can be measured without knowing or assuming anything about the rotational axis of the distal object. Consequently, if the $x y$ rotational axis of the distal object and the rotational component $\dot{q}$ are found by a smallangle process operating close to the fixation point, the depth-related quantity $\dot{\alpha} D$ can be estimated for any point $n$ on the projection surface simply by measuring the 

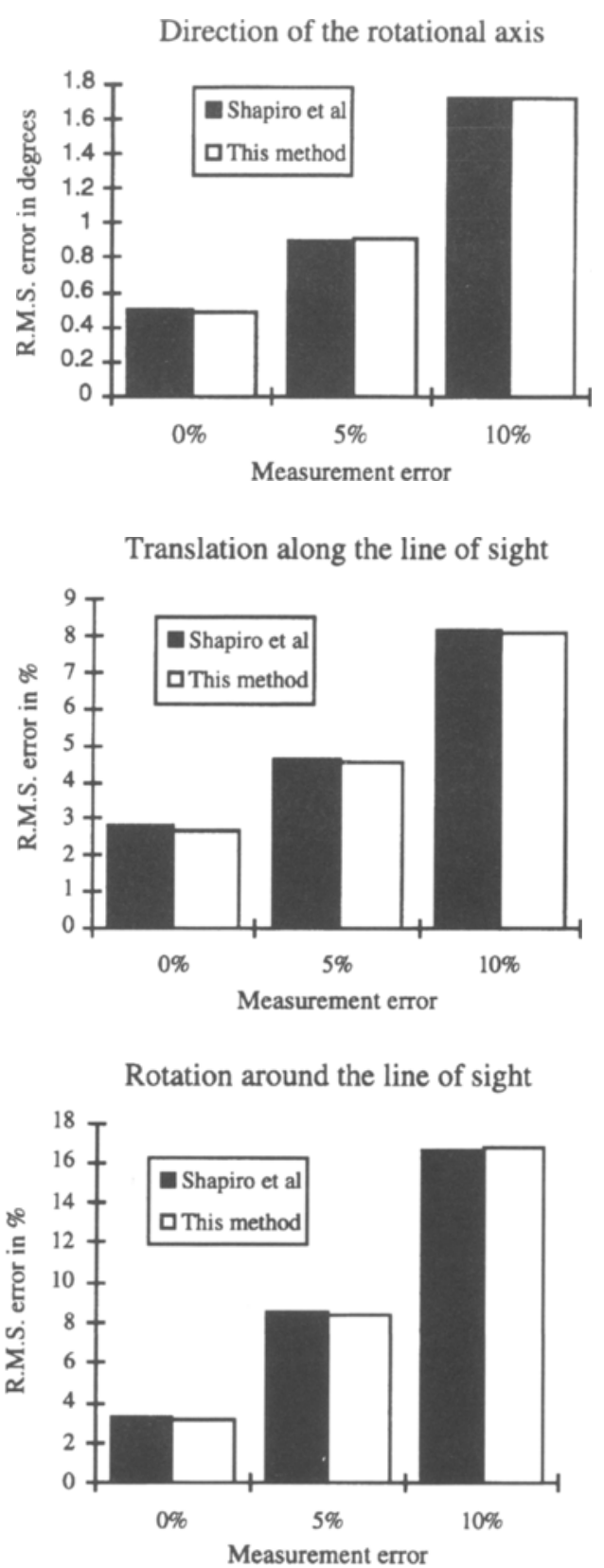

Figure 5. Examples of the results of the numerical comparisons between the Shapiro et al. algorithm and the proposed smallangle process using synthetic optic-flow fields produced by the use of noncoplanar, polar-projected landmarks (see Appendix B). The conditions illustrated are visual angle $=4^{\circ} ;(\Delta z, \Delta \alpha)=$ $(0.0071,0.0071)$; internal depth of distal object $=10 \%$; and all three error levels $=0 \%, 5 \%$, and $10 \%$. Each bar represents RMS error values gathered from 500 runs.

quantity $r_{n}\left(\dot{\theta}_{n}-\dot{q}\right) / \sin \left(\theta_{n}\right)$, where $\theta_{n}$ is measured in the coordinate system chosen by the small-angle process. That is,

$$
\dot{\alpha} D_{n}=\frac{r_{n}\left(\dot{\theta}_{n}-\dot{q}\right)}{\sin \left(\theta_{n}\right)}
$$

A drawback of this equation is that it cannot operate where $\theta$ is close to zero, (i.e., close to the $x$-axis). This can be overcome by using Equation 8 directly. The relative simplicity of Equation 13 is lost, but affine depth values can be found for all points on the projection surface. If, like before, the $x y$ rotational axis of the distal object and the parameters $\dot{q}$ and $\dot{z}$ are found by the small-angle part of the algorithm operating close to the fixation point, the distance $D$ can be expressed in terms of an unknown value of $\dot{\alpha}$ for any point on the projection surface:

$$
D_{n}=\frac{\left(\dot{x}_{n}-x_{n} \dot{z}+y_{n} \dot{q}-x_{n}^{2} \dot{\alpha}\right)}{\left(x_{n} \dot{z}-\dot{\alpha}\right)}
$$

4.7.2. Estimating the remaining unknown parameter of relative motion. The term $y D(x, y)$ in Equation 8 can be expressed as
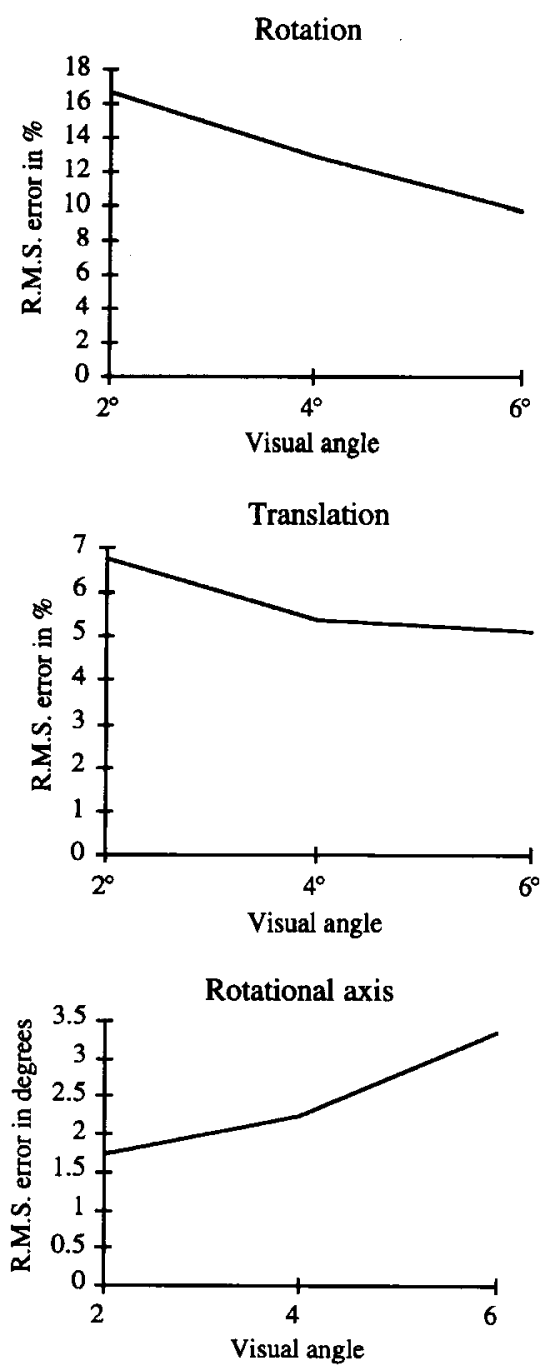

Figure 6. Results illustrating opposite tendencies in errors produced as a function of visual angle. The conditions illustrated are visual angle $=4^{\circ} ;(\Delta z, \Delta \alpha)=(0.0071,0.0071) ;$ error level $=$ $10 \%$; and all three visual angles $=2^{\circ}, 4^{\circ}$, and $6^{\circ}$. 


$$
y D(x, y)=\frac{1}{\dot{\alpha}}(x \dot{y}-y \dot{x})-\frac{\dot{q}}{\dot{\alpha}}\left(x^{2}+y^{2}\right) .
$$

Using this in Equation 8 gives Equation 15:

$$
\dot{y}=x y \dot{\alpha}+x \dot{q}+\dot{y} z+\frac{\dot{z}}{\dot{\alpha}}(x \dot{y}-y \dot{x})-\frac{\dot{q} \dot{z}}{\dot{\alpha}}\left(x^{2}+y^{2}\right) \text {. }
$$

This equation expresses a relationship between the parameters of relative motion and measurable quantities on the projection surface that are independent of the depth values of the viewed landmarks. As before, the $x y$ rotational axis is considered to be the $y$-axis.

If the $x y$ rotational axis is recovered by the small-angle processes described above (and, thus, the existence of a nonzero value of $\dot{\alpha}$ ), several methods are conceivable to estimate the remaining unknown parameter $\dot{\alpha}$ using the relationship expressed by Equation 15 . The difference between these methods is the amount of information being "carried over" into the wide-angle process from the smallangle process.

One option is to use all the estimates produced by the small-angle process. Only $\dot{\alpha}$ is then left to be estimated by the wide-angle process. Equation 15 can be written

$$
y-(x \dot{q}+y \dot{z})=x y \dot{\alpha}+\frac{1}{\dot{\alpha}}\left[\dot{z}(x \dot{y}-y \dot{x})-\dot{z} \dot{q}\left(x^{2}+y^{2}\right)\right] .
$$

The parameter $\dot{\alpha}$ can thus be found by solving a quadratic equation for any point in the periphery. However, in the presence of measurement errors, more efficient methods are conceivable. Equation 16a can be transformed into a well-behaved error function:

$$
\begin{aligned}
\mathrm{e}(\dot{\alpha})=\Sigma & \|\left(\dot{y}_{i}-x_{i} \dot{q}-y_{i} \dot{z}\right) \\
& \left.-\left\{x_{i} y_{i} \dot{\alpha}+\frac{1}{\dot{\alpha}}\left[\dot{z}\left(x_{i} \dot{y}_{i}-y_{i} \dot{x}_{i}\right)-\dot{z} \dot{q}\left(x_{i}^{2}+y_{i}^{2}\right)\right]\right\}\right]^{2}
\end{aligned}
$$

and can be examined by a simple steepest descent method. No claim is made that Equation $16 \mathrm{~b}$ describes the best way of finding $\dot{\alpha}$ given these circumstances. However, it is good enough to illustrate the viability of the general approach, as is demonstrated below.

Two minima usually exist when Equation $16 \mathrm{~b}$ is used: one negative and one positive. The error function, therefore, has to be evaluated on each side of zero and the lowest of the two minima chosen as the global minimum. These two minima may have perceptual counterparts. In that the $\dot{\alpha}$ values they represent usually are of approximately the same magnitude and have opposite signs, using them in the 3-D reconstruction will result in two 3-D objects rotating in opposite directions and with inverted 3-D structures-the Necker-type inversion often found with moving dot stimuli. Because, at least when perspective projection and large visual angles are used, one of the minima is larger than the other, a greater sensation of nonrigidity could accompany the utilization of this larger value.

The results achieved when using this approach to finding $\dot{\alpha}$ are illustrated in the top panel of Figure 7. As can be seen, the results are encouraging, although the root mean square error levels cannot be taken too literally. They will depend on the number of landmarks and the situation facing the small-angle process. However, they indicate that this type of process could be useful to a visual system.

Another option for the use of wide-angle input is to use only the estimate of the direction of the $x y$ rotational axis from the small-angle process and to let the wide-angle process refine the estimates of $\dot{q}$ and $\dot{z}$ along with finding an estimate of $\dot{\alpha}$. This can be done in at least two different ways. One is to use all constraints inherent in the problem and find the values by a nonlinear search procedure. The other is to regard Equation 15 as a linear function with five unknown parameters and use multiple regression to determine these unknown parameters.

The nonlinear approach was tried by rearranging Equation 15 into an error function. Good initial values for the $\dot{q}$ and $\dot{z}$ parameters are given by the small-angle process, but it is difficult to assess a good initial value for $\dot{\alpha}$. Because of this, the nonlinear search method used (the MATLAB fmins procedure) was sometimes plagued by the existence of local minima and nonconvergence. Such phenomena are common also when the whole M\&SFM problem is being solved in its entirety by nonlinear search methods (e.g., Heeger \& Jepson, 1992; Jerian \& Jain, 1991; Pradzny, 1980).

The pseudolinear approach, treating Equation 15 as linear, means increasing the degrees of freedom to gain computational ease. A similar strategy to solving the entire M\&SFM problem in the polar-perspective case was first put forward by Longuet-Higgins (1981) and is discussed in Heeger and Jepson (1992). Heeger and Jepson conclude that such methods are too sensitive to measurement errors to be practically useful. In this case, however, when the $x y$ rotational axis is known (i.e., approximately recovered by the small-angle weak-perspective process) and the visual angle is large, this sensitivity is not generally present. Given that the landmarks have sufficient variations in depth values and that these depth values are nonlinearly related to their projected positions on the projection surface, the pseudolinear approach works extremely well. However, when the depth values of the landmarks are linearly related to their projected positions on the projection surface, as in the "floor-ceiling" scene used in the simulations, the size of the errors produced by adding measurement errors casts some doubts on its usefulness to a visual system. There is one exception to this finding, of course. When the viewed landmarks belong to a plane that is perpendicular to the line of sight, the linear relationship between depth values and projected positions does not pose any problems. The results of using 

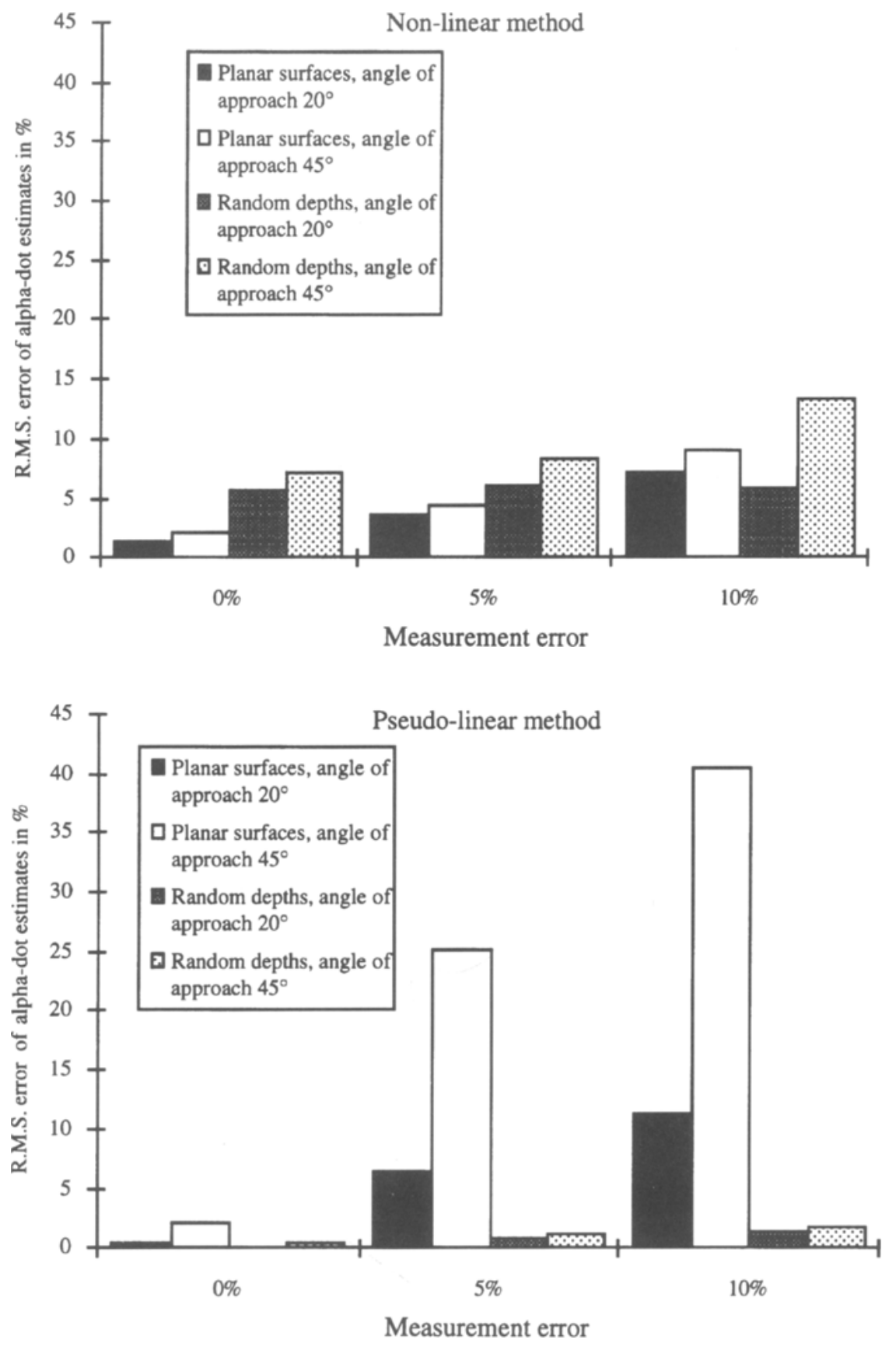

Figure 7. The results of the numerical experiments performed to evaluate how well $\dot{\alpha}$ can be estimated by different methods operating on input defined over large visual angles. The results from the two most successful methods are shown: the nonlinear method estimating only $\dot{\alpha}$, and the pseudolinear method estimating all parameters of relative motion except $\delta$.

the pseudolinear approach are illustrated in the bottom panel of Figure 7.

The wide-angle process that best models the performance of the human visual system cannot be assessed given the presently available psychophysical evidence. The main point is, however, that a weak-perspective small-angle process can be complemented by a polar-projection -based wide-angle process and function well.

\section{SPATIAL DERIVATIVES REVISITED}

Most M\&SFM methods are primarily SFM methods - that is, they are designed to reconstruct the 3-D layout of a viewed rigid scene. The recovery of the relative motion is mostly an unavoidable, although interesting, by-product. In the case of planar surfaces engaged in unrestricted motion and seen at small visual angles, a 3-D 
recovery is not generally possible. Because of this, no attempts are made by most M\&SFM methods to recover information about the relative motion in such cases.

The human visual system, however, is very sensitive to optic flow that can be interpreted as caused by moving planar surfaces, and such optic-flow patterns are frequently used in studies of the perception of visual motion. As a result, the relation between studies on human sensitivity to visual motion and the possibilities offered by M\&SFM processes is somewhat unclear. This is illustrated by studies concerned with the sensitivity of the human visual system to spatial derivatives of optic flow (cf. Section 2.1.3).

The spatial derivatives of optic flow in a small area around a point on a projection surface constitute a description of the flow at that point. They are thus measures of proximal events. In contrast, components of relative motion ( $\dot{z}, \dot{q}, \dot{\alpha}$, and $\delta$ in the present terminology) between an observer and a viewed object, which are recovered by an M\&SFM process, are distal events. The spatial derivatives of optic flow are usually split into components commonly referred to as divergence, rotation, and deformation (Koenderink \& van Doorn, 1976). In the case of optic flow caused by moving rigid objects, each of these (proximal) components of spatial derivatives is dependent upon two or three of the (distal) components of relative motion $(\dot{z}, \dot{q}, \dot{\alpha}$, and $\delta)$ in addition to the $3-\mathrm{D}$ orientation of the viewed surface.

One case exists, however, when the relation between the components of spatial derivatives and the components of relative motion is more straightforward: When the viewed object is a (planar) rigid surface orthogonal to the line of sight and the proximal area considered is close to the fixation point, then no deformation exists, the divergence is proportional to the value of $\dot{z}$, and the rotation is proportional to $\dot{q}$.

In contrast to most other M\&SFM methods, the one presented in this paper can discover the presence of this situation and estimate the values of the parameters $\dot{z}$ and $\dot{q}$. Thus, this method will appear as if it is estimating the divergence and the rotation, when, in fact, it is estimating the distal parameters $\dot{z}$ and $\dot{q}$.

Consequently, proximal motion patterns exhibiting pure divergence (pure expansion or contraction), pure rotation, or combinations of such patterns (spiral-like motions) will be interpreted by the proposed M\&SFM mechanism, and estimates of the relative distal motion will be produced. These estimates will also be proportional to the values of the divergence and the rotation.

In terms of psychophysical experimentation, this means that the sensitivity of human subjects to divergence and rotation when pure divergence, pure rotation, or combinations thereof are used (e.g., Ahlström \& Börjesson, in press; De Bruyn \& Orban, 1990, 1993; Freeman \& Harris, 1992; Reagan \& Beverley, 1978) cannot be taken as evidence that the human visual system is sensitive to these components of spatial derivatives as such. It could instead be operating in a way related to the M\&SFM mechanism proposed in this paper. A critical experiment, which could distinguish between a proximal (spatial derivatives) analysis and a distal (components of relative motion) analysis by the human visual system, would involve the use of proximal motion patterns where the values of all components of the spatial derivatives are simultaneously nonzero and systematically varied.

The similarity in output in certain cases by the M\&SFM mechanism proposed in this paper and a mechanism sensitive to divergence and rotation is present only in an area close to the fixation point. However, this is consistent both with the findings on human sensitivity to pure divergence and rotation cited above and with the findings of Braddick and Holliday (1992) described in Section 2.1.3.

\section{DISCUSSION}

The M\&SFM process described in this paper is designed to deal with optic flow produced by rigidly connected landmarks moving relative to the observer. It produces an output that is similar to the performance of human observers, when faced with such flow, as it is reflected in the literature. However, to fully evaluate the limits of this similarity, a number of psychophysical studies are needed. The study suggested in Section 5, proposed to examine whether spatial derivatives are really estimated by the human visual system, is an example. Another is the possibility, opened by the present approach, to have nonuniformly distributed coordinate systems in the small-angle process. This would lead to a varied resolution in the recovery of the $x y$ rotational axis. One conceivable use of such a nonuniform distribution would be to have the coordinate systems closer together in the vicinities of the horizontal and vertical axes of the eye. During ego-motion, the $x y$ rotational axis is usually close to the vertical axis or, if we are only moving up and down, close to the horizontal axis. This arrangement of coordinate systems would lead to an "oblique-like" effect where the sensitivity to the directions of $x y$ rotational axes is lower at "oblique" directions.

As is clear from the previous sections, there are several important aspects of perceiving motion and depth from visual motion that were not addressed. These include continuous (in time) optic flow, how several independently moving objects are handled, and how the flow fields produced by nonrigid objects are analyzed.

These and other aspects are possible to include in the present framework and in several different ways. However, in that the goal of the present work is to better understand human M\&SFM capabilities, more psychophysical data are needed to guide the way before this is undertaken.

\section{REFERENCES}

Ahlström, U. B., \& BöRJesson, E. (in press). Segregation of motion structure from random visual noise. Perception.

BörJEsSON, E., \& LIND, M. (1996). The effect of polar projection on the perception of euclidean structure from motion. Perception \& Psychophysics, 58, 871-882.

BörJesson, E., \& von Hofsten, C. (1973). Visual perception of motion in depth: Application of a vector model to three-dot motion patterns. Perception \& Psychophysics, 13, 169-179. 
BörJesson, E., \& von Hofsten, C. (1975). A vector model for perceived object rotation and translation in depth. Psychological Research, 38, 209-230.

BRADDICK, O. J., \& Holliday, I. E. (1992). Serial search for targets defined by divergence or deformation of optic flow. Perception, 20, 345-354.

De Bruyn, B., \& Orban, G. A. (1990). The role of direction information in the perception of geometric optic flow components. Perception \& Psychophysics, 47, 433-438.

De BruYn, B., \& OrBan, G. A. (1993). Segregation of spatially superimposed optic flow components. Journal of Experimental Psychology: Human Perception \& Performance, 19, 1014-1027.

Dosher, B. A., LANDY, G., \& SPERLING, G. (1989). Kinetic depth effect and optic flow: I. 3-D shape from fourier motion. Vision Research, 29, 1789-1813.

Faugeras, O. (1993). Three-dimensional computer vision: A geometric viewpoint. Cambridge, MA: MIT Press.

Freeman, T. C. A., \& Harris, M. G. (1992). Human sensitivity to expanding and rotating motion: Effects of complementary masking and directional structure. Vision Research, 32, 81-87.

GiBson, J. J. (1950). The perception of the visual world. Boston: Houghton Mifflin.

GIBSon, J. J. (1966). The senses considered as perceptual systems. Boston: Houghton Mifflin.

GORDON, D. A. (1965). Static and dynamic visual fields in human space perception. Journal of the Optical Society of America, 55, 1296-1303.

HEEGER, D. J., \& JEPSON, A. D. (1992). Subspace methods for recovering rigid motion: I. Algorithm and implementation. International Journal of Computer Vision, 7, 95-117.

Husain, M., Treue, S., \& Andersen, R. A. (1989). Surface interpolation in three-dimensional structure-from-motion perception. Neural Computation, 1, 324-333.

JeRIAN, C. P., \& JAIN, R. J. (1993). Structure from motion: A critical analysis of methods. IEEE Transactions on Systems, Man, \& Cybernetics, 21, 572-588.

KoEnderinK, J. J. (1986). Optic flow. Vision Research, 26, 161-180.

Koenderink, J. J. (1990). Some theoretical aspects of optic flow. In R. Warren \& A. H. Wertheim (Eds.), Perception and control of selfmotion (pp. 53-68). Hillsdale, NJ: Erlbaum.

KoENDERINK, J. J., \& VAN DOORN, A. (1976). Local structure of movement parallax of the plane. Journal of the Optical Society of America, 66, 717-723.

Koenderink, J. J., \& van DoORn, A. (1987). Facts on optic flow. Biological Cybernetics, 56, 247-254.

KoENDERINK, J. J., \& VAN DOORN, A. (1991). Affine structure from motion. Journal of the Optical Society of America A, 8, 377-385.

Landy, G., Dosher, B. A., Sperling, G., \& Perkins, M. E. (1991). The kinetic depth effect and optic flow: II. First and second order motion. Vision Research, 31, 859-876.

LAPPIN, J. S., \& AHLSTRÖM, U. B. (1994). On the scaling of visual space from motion-In response to Pizlo and Salach-Golyska. Perception \& Psychophysics, 55, 235-242.

LAPPIN, J. S., \& Love, S. R. (1992). Planar motion permits perception of metric structure in stereopsis. Perception \& Psychophysics, 51, 86-102.

LEE, D. N. (1974). Visual information during locomotion. In R. B. Macleod \& H. L. Pick (Eds.), Perception: Essays in honour of James J. Gibson (pp. 250-267). Ithaca, NY: Cornell University Press.

Lind, M., \& BörJesson, E. (1996). Perceived structure from two successive polar projected views: Consistent versus variable mapping of 3-D Euclidean structure. Manuscript in preparation.

Longuet-Higgins, H. C. (1981). A computer algorithm for reconstructing a scene from two projections. Nature, 293, 133-135.

Longuet-Higgins, H. C., \& PradzNy, K. (1980). The interpretation of a moving retinal image. Proceedings of the Royal Society of London: Series $B, \mathbf{2 3 3}, 385-397$.

MaYBANK, S. (1993). Theory of reconstruction from image motion. Berlin: Springer-Verlag.

MCKeE, S. P., \& WelCh, L. (1985). Sequential recruitment in the discrimination of velocity. Journal of the Optical Society of America A, 2, 243-251.

NaKAYAMA, K. (1985). Biological image processing: A review. Vision Research, 25, 625-660.
NaKaYama, K., \& LoOmis, J. M. (1974). Optical velocity patterns, velocity-sensitive neurons and space perception: A hypothesis. Perception, 3, 63-80.

Nakayama, K., \& Tyler, C. W. (1981). Psychophysical isolation of movement sensitivity by removal of familiar position cues. Vision Research, 21, 427-433.

Norman, J. F., \& ToDD, J. T. (1993). The perceptual analysis of structure from motion for rotating objects undergoing affine stretching transformations. Perception \& Psychophysics, 53, 279-29l.

Pizlo, Z., \& Salach-Golyska, M. (1994). Is vision metric? Comment on Lappin and Love (1992). Perception \& Psychophysics, 55, 230-234.

PradzNy, K. (1980). Egomotion and relative depth map from optical flow. Biological Cybernetics, 36, 87-102.

ReaGan, D., \& Beverley, K. I. (1978). Looming detectors in the human visual pathway, Vision Research, 18, 415-421.

ROGERS, B. J. (1993). Motion parallax and other dynamic cues for depth in humans. In F. A. Miles \& J. Wallman (Eds.), Visual motion and its role in the stabilization of gaze (pp. 119-137). Amsterdam: Elsevier.

Shapiro, L. S., Zisserman, A., \& Brady, M. (1995). 3-D motion recovery via affine epipolar geometry. International Journal of Computer Vision, 16, 147-182.

Todd, J. T., Akerstrom, R. A., Reichel, F. D., \& Hayes, W. (1988). Apparent rotation in three-dimensional space: Effects of temporal, spatial, and structural factors. Perception \& Psychophysics, 43, 179-188.

TODD, J. T., \& Bressan, P. (1990). The perception of 3-dimensional affine structure from minimal apparent motion sequences. Perception \& Psychophysics, 48, 419-430.

ToDD, J. T., \& Norman, J. F. (1991). The visual perception of smoothly curved surfaces from minimal apparent motion sequences. Perception \& Psychophysics, 50, 509-523.

Tresilian, J. R. (1993). Four questions of time to contact: A critical examination of research on interceptive timing. Perception, 22, 653-680.

\section{NOTES}

1. A systematic description of various projection types ("cameras") and their properties in relation to M\&SFM processes can be found in Shapiro et al. (1995).

2. These authors (see, e.g., Koenderink, 1986) have also proposed sensing mechanisms of the deformation component that are not based on retinal velocities as such. In light of the discussion on the input to the M\&SFM process, however, this is not relevant in the present context.

3. The use of higher order spatial derivatives has been proposed (e.g., Longuet-Higgins \& Pradzny, 1980).

4. This is true unless some a priori constraint can be used-for instance, knowledge that the surrounding world is of a known shape or of a shape having fewer degrees of freedom than the number of available velocity estimates.

5. This is a property that algorithms based on weak perspective share with algorithms based on orthographic projection. It is usually dealt with by letting the algorithms analyze more than two views.

6. Similar notions have been used by authors other than Koenderink and van Doorn (1987)-for example, by Gordon (1965), Lee (1974), Nakayama and Loomis (1974), and Pradzny (1980).

7. Of special interest in this case is the inverse of the value of $\dot{z}$; it represents "time to contact" (Lee, 1974; Tresilian, 1993).

\section{APPENDIX A}

Equation numbers below are the same as equation numbers in text.

Please refer to Figure 2.

A distal point $(X, Y, Z)$ is projected onto the $x y$ plane using $(z=1)$ as the projection point. The projection of the point is at $(x, y)$. The following relationships hold:

$$
x=\frac{X}{(1-Z)}
$$

and 


$$
y=\frac{Y}{(1-Z)} .
$$

\section{Rotation Around the $\boldsymbol{z}$-Axis}

Polar coordinates on the projection surface are used:

$$
\left\{\begin{array}{l}
x=r \cos (\theta) \\
y=r \sin (\theta)
\end{array} .\right.
$$

Their derivatives with respect to time in this case are

$$
\left\{\begin{array}{l}
\dot{x}=-r \sin (\theta) \dot{\theta} \\
\dot{y}=r \cos (\theta) \dot{\theta}
\end{array}\right.
$$

Since $\dot{q}=\dot{\theta}$, by definition this can be written as

$$
\left\{\begin{array}{l}
\dot{x}=-y \dot{q} \\
\dot{y}=x \dot{q}
\end{array}\right.
$$

\section{Translation of the Object Along the $z$-Axis}

The derivative with respect to time of the $x$-coordinate, in this case, is

$$
\dot{x}=\frac{X \dot{z}}{(1-Z)^{2}},
$$

which can be written as

$$
\dot{x}=x \dot{z} \frac{1}{(1-Z)} .
$$

Since

$$
\frac{1}{(1-Z)}=1+\frac{Z}{(1-Z)},
$$

and, by definition

$$
D=\frac{Z}{(1-Z)},
$$

this means that

$$
\dot{x}=x \dot{z}+x \dot{z} D .
$$

Consequently, the derivative with respect to time of the $y$-coordinate can be expressed as

$$
\dot{y}=y \dot{z}+y \dot{z} D .
$$

\section{Rotation of the Object Around the $\boldsymbol{y}$-Axis}

The point $(X, Y, Z)$ is first expressed in cylindrical coordinates $(Y, p, \alpha)$, such that

$$
\left\{\begin{array}{l}
X=p \cos (\alpha) \\
Y=Y \\
Z=p \sin (\alpha)
\end{array} .\right.
$$

The derivatives with respect to time, in this case, can be written

$$
\left\{\begin{array}{l}
\dot{X}=-p \sin (\alpha) \dot{\alpha} \\
\dot{Y}=0 \\
\dot{Z}=p \cos (\alpha) \dot{\alpha}
\end{array}\right.
$$

which means that

$$
\left\{\begin{array}{l}
\dot{X}=-Z \dot{\alpha} \\
\dot{Y}=0 \\
\dot{Z}=X \dot{\alpha}
\end{array} .\right.
$$

The derivatives with respect to time of the projection of the point on the $x y$ plane, in this case, are

$$
\left\{\begin{array}{l}
\dot{x}=\frac{\dot{X}}{(1-Z)}+\frac{X \dot{Z}}{(1-Z)^{2}} \\
\dot{y}=\frac{Y \dot{Z}}{(1-Z)^{2}}
\end{array} .\right.
$$

Using the expression derived from the cylindrical coordinates, this can be written as

$$
\left\{\begin{array}{l}
\dot{x}=\frac{-Z \dot{\alpha}}{(1-Z)}+\frac{X^{2} \dot{\alpha}}{(1-Z)^{2}} \\
\dot{y}=\frac{Y X \dot{\alpha}}{(1-Z)^{2}}
\end{array},\right.
$$

which in turn can be simplified as

$$
\left\{\begin{array}{l}
\dot{x}=x^{2} \dot{\alpha}-D \dot{\alpha} \\
\dot{y}=x y \dot{\alpha}
\end{array}\right. \text {. }
$$

\section{Examining the Quantity $y D(x, y)$}

Combining Equations 7 and 8 (see text, p. 1092) yields

$$
\frac{\dot{y}}{y}-\frac{\dot{x}}{x}=\frac{\dot{\alpha} D}{x}+\dot{q}\left(\frac{x}{y}+\frac{y}{x}\right)
$$

which can be written as

$$
D=\frac{1}{\dot{\alpha}}\left(\dot{y} \frac{x}{y}-\dot{x}\right)-\frac{\dot{q}}{\dot{\alpha}}\left(\frac{x^{2}}{y}+y\right) .
$$

Consequently,

$$
y D=\frac{1}{\dot{\alpha}}(x \dot{y}-y \dot{x})-\frac{\dot{q}}{\dot{\alpha}}\left(x^{2}+y^{2}\right) .
$$

Then, $(x, y)$ and $(\dot{x}, \dot{y})$ are expressed in terms of polar coordinates on the projection surface

$$
\begin{aligned}
& \left\{\begin{array}{l}
x=r \cos (\theta) \\
y=r \sin (\theta)
\end{array}\right. \\
& \left\{\begin{array}{l}
\dot{x}=\dot{r} \cos (\theta)-r \sin (\theta) \dot{\theta} \\
\dot{y}=\dot{r} \sin (\theta)+r \cos (\theta) \dot{\theta}
\end{array}\right.
\end{aligned}
$$

This means that

$$
x \dot{y}=r \dot{r} \sin (\theta) \cos (\theta)+r^{2} \cos ^{2}(\theta) \dot{\theta}
$$

and

$$
y \dot{x}=r \dot{r} \sin (\theta) \cos (\theta)-r^{2} \sin ^{2}(\theta) \dot{\theta}
$$

and that

$$
(x \dot{y}-y \dot{x})=r^{2} \dot{\theta} .
$$

By definition, $x^{2}+y^{2}=r^{2}$.

To find an expression in terms of polar coordinates of the term $\dot{\alpha} D$ when $\dot{q}$ is zero, we note 


$$
\dot{\alpha} D=x\left(\frac{\dot{y}}{y}-\frac{\dot{x}}{x}\right),
$$

which, in terms of polar coordinates, is

$$
\dot{\alpha} D=\dot{\theta} r \cos (\theta)\left(\frac{\cos (\theta)}{\sin (\theta)}+\frac{\sin (\theta)}{\cos (\theta)}\right)
$$

and can be expressed as

$$
\dot{\alpha} D=\frac{r \dot{\theta}}{\sin (\theta)} .
$$

\section{APPENDIX B \\ How the Numerical Evaluations Were Performed}

The evaluations were regarded as numerical experiments. Some independent variables were systematically varied, and others were controlled by randomly drawing values, at runtime, from a specified distribution. Polar projection was used throughout the evaluations.

\section{B1. THE SMALL-ANGLE PROCESS}

\section{B1.1. Systematically Varied Independent Variables}

B1.1.1. Visual angles. Three different visual angles were investigated: $2^{\circ}, 4^{\circ}$, and $6^{\circ}$. The visual angle was considered to express the diameter of a circular area on the projection surface centered around the line of sight.

B1.1.2. Internal depth of the synthetic distal objects. Two levels of maximum internal depths were chosen: $10 \%$ and $30 \%$ of the distance between the fixated landmark and the projection point. The depth value used for each point was drawn randomly from a uniform distribution between -0.05 and 0.05 in the $10 \%$ condition and between -0.15 and 0.15 in the $30 \%$ condition.

B1.1.3. $\Delta z$ and $\Delta \alpha$. The translation along the line of sight $(\Delta z)$ and the amount of rotation around the $x y$ rotational axis $(\Delta \alpha)$ was set to one of three combinations: $(\Delta z, \Delta \alpha)=(0.0094$, $0.0034) ;(\Delta z, \Delta \alpha)=(0.0071,0.0071) ;$ and $(\Delta z, \Delta \alpha)=(0.0034$, $0.0094) . \Delta z$ was measured in units defined by the distance to the fixation point, which by definition is unity, and $\Delta \alpha$ was measured in radians. If an ego-motion description of these combinations is used, the three conditions correspond to an angle of approach to the object of $70^{\circ}, 45^{\circ}$, and $20^{\circ}$, respectively.

B1.1.4. Measurement error. Measurement error was added by, for each landmark and run, randomly selecting an error vector. The magnitude of the error vector was drawn from a normal distribution having zero as its mean and $0 \%, 5 \%$, or $10 \%$, depending on the error level, of the magnitude of the landmark's true velocity vector as its variance. The direction of the error vector was drawn from a uniform distribution of directions between $0^{\circ}$ and $360^{\circ}$.

B1.1.5. Design. These factors define a $3 \times 2 \times 3 \times 3$ experiment yielding 54 conditions. In each condition, 500 runs were performed, and the resulting flow fields analyzed by means of both the Shapiro et al. (1995) algorithm and the small visualangle process.

\section{B1.2. Random Factors}

B1.2.1. Number of landmarks. All flow fields were produced by randomly choosing between 7 and 30 landmarks from a uniform distribution before each run.
B1.2.2. Position of landmarks. The landmarks were assigned distal positions by first randomly choosing their projected positions on the projection surface from uniform distributions within the limits set by the visual angle condition. The projection point and the randomly chosen projected positions defined straight lines in $(x, y, z)$ space, one for each landmark, along which the distal landmarks could be positioned. Then, $z$-values were randomly drawn within the limits set by the internal depth condition. The values obtained by this procedure determined the distal positions.

B1.2.3. $\Delta q$ and $\delta$. The amount of rotation around the line of sight $(\Delta q)$ was randomly drawn from a uniform distribution between -0.007 radians and +0.007 radians $\left( \pm 0.4^{\circ}\right)$, and the direction of the $x y$ rotational axis $(\delta)$ was randomly drawn from a uniform distribution between $0^{\circ}$ and $180^{\circ}$. This was performed before each run.

\section{B2. THE WIDE-ANGLE EVALUATIONS OF $\Delta \alpha$}

The wide-angle evaluation was always preceded by an analysis by the small-angle process on landmarks that projected within a circular area centered around the line of sight with a diameter of $5^{\circ}$ of visual angle. In all conditions, 30 landmarks were used that projected to random positions within this circular area. The distal positions of these landmarks were determined as described in Section B1.2.2 using a maximum depth value of $10 \%$.

Outside of the above-mentioned circular area, another circular area with a diameter of $140^{\circ}$ of visual angle was defined. Within this area, but outside of the inner circular area, another 200 positions were randomly drawn from uniform distributions before each run. Using the procedure described in Section B1.2.2, and with limits set by the object definitions described below, the distal positions of these additional 200 landmarks were then determined.

The direction of the $x y$ rotational axis $(\delta)$ was always kept at $90^{\circ}$.

\section{B2.1. Systematically Varied Independent Variables}

B2.1.1. Internal depth of the synthetic distal objects. Two different types of distal objects were defined. One consisted of randomly assigned depth values to the landmarks from a uniform distribution, resulting in a maximum internal depth of $60 \%$ of the distance between the projection point and the fixated point. The other was a room-like structure with a floor extending from below the fixation point to below the fixated point, a far wall orthogonal to the floor, and a ceiling extending from the wall and above the projection point. The ceiling was parallel to the floor.

B2.1.2. $\Delta z$ and $\Delta \alpha$. Two of the three sets of values defined in the evaluation of the small-angle process were used. They correspond to an angle of approach to the object of $20^{\circ}$ and $45^{\circ}$ if an ego-motion view of the relative motion is used.

B2.1.3. Measurement error. Measurement error was added in the same way as in the evaluation of the small-angle process.

B2.1.4. Design. These factors define a $2 \times 2 \times 3$ experiment yielding 12 conditions. In each condition, 200 runs were performed, and the resulting flow fields were analyzed by the three methods described in the text: the pseudolinear method solving for all parameters of relative motion except for $\delta$, a nonlinear method solving only for $\Delta \alpha$, and a nonlinear method solving for all parameters of relative motion except for $\delta$.

(Manuscript received June 6, 1994; revision accepted for publication January 21,1996 .) 